\title{
Portal vein thrombosis in liver transplantation: radiologic evaluation, risk factors, and occult diagnosis
}

\author{
Adam Hauch ${ }^{1}$, Carl Winkler ${ }^{1}$, Eric Katz ${ }^{1}$, Peter W. Lundberg ${ }^{1}$, Mary Killackey ${ }^{1}$, Anil S. \\ Paramesh $^{1}$, Luis A. Balart ${ }^{2}$, Nathan J. Shores ${ }^{2}$, Martin Moehlen ${ }^{2}$, Ward Miller ${ }^{2}$, Douglas P. \\ Slakey $^{1}$, Joseph F. Buell ${ }^{1}$, Bob H. Saggi ${ }^{1}$ \\ ${ }^{1}$ Tulane Transplant Institute, Tulane University School of Medicine, New Orleans, LA 70112, USA \\ ${ }^{2}$ Department of Gastroenterology, Section of Hepatology, Tulane University School of Medicine, New Orleans, LA 70112, USA
}

\begin{abstract}
Aim: Portal vein thrombosis (PVT) in the liver transplant recipient poses many challenges. Unfortunately, the risk factors and effects on outcomes of PVT are not well-defined. Methods: This study analyzed the experience with PVT in liver transplant program from 2007 to 2013. This included the effectiveness of PVT diagnostics and its risk factors using logistical regression. The primary endpoints were Kaplan-Meir patient and graft survival. The secondary endpoints were the length of stay (LOS), transfusion rate, and overall morbidity. Independent predictors of survival were identified using a Cox's proportional hazards model. Results: Two hundred and sixteen consecutive liver transplant recipients were examined, and 30 (13.8\%) had either a total or partial PVT. Two hundred and five patients had imaging within 1 year of liver transplantation with only 7 (23.3\%) of the 30 PVTs identified pre-operatively. Calculated sensitivity (4.8-50\%) and negative predictive values (10.5-22.2\%) were poor. Only, age significantly predicted PVT $[P=0.037 /$ hazard ratio $(H R)=0.95]$. Ninety-day-patient and graft survival for PVT was similar at 6 months, although 1-year survival was significantly lower. "Occult" PVT was not associated with inferior survival. Model for end-stage liver disease score $>$ $25(P=0.001, \mathrm{HR}=0.49 / P=0.004, \mathrm{HR}=0.52)$ and age $>60$ years $(P=0.017, \mathrm{HR}=0.64 / P=0.013, \mathrm{HR}=0.67)$ were significant predictors of patient and graft survival. Although the transfusion rate was significantly greater with PVT, LOS, and morbidity were not. Conclusion: Older recipients had a greater likelihood of PVT. Diagnostic studies were not effective at excluding PVT, and occult diagnosis did not affect the outcome. PVT was not an independent predictor of mortality or graft loss, but was associated with greater blood loss but not increased LOS or morbidity.
\end{abstract}

Key words: Advanced age; graft survival; patient survival; post-transplant outcomes; resource utilization

Address for correspondence:

Dr. Adam Hauch, Tulane Transplant Institute, Tulane University School of Medicine, 1415 Tulane Ave. HC-5, New Orleans, LA 70112, USA. E-mail: ahauch@tulane.edu

Received: 23-04-2015, Accepted: 06-09-2015

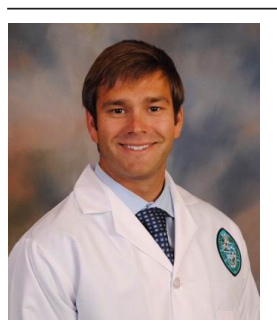

Adam Hauch received his undergraduate degree in Mathematics at Northwestern University. He then went on to receive his medical and business degrees from the University of Southern California. He is currently in his fourth clinical year of general surgery residency at Tulane University where he is also actively involved in both clinical and basic science research.

\begin{tabular}{|l|c|}
\hline \multicolumn{3}{|c|}{ Access this article online } \\
\hline \multirow{2}{*}{ Website: } & Quick Response Code \\
\cline { 2 - 2 } http://www.hrjournal.net/ & \\
\hline &
\end{tabular}

This is an open access article distributed under the terms of the Creative Commons Attribution-NonCommercial-ShareAlike 3.0 License, which allows others to remix, tweak, and build upon the work non-commercially, as long as the author is credited and the new creations are licensed under the identical terms.

For reprints contact: service@oaepublish.com

How to cite this article: Hauch A, Winkler C, Katz E, Lundberg PW, Killackey M, Paramesh AS, Balart LA, Shores NJ, Moehlen M, Miller W, Slakey DP, Buell JF, Saggi BH. Portal vein thrombosis in liver transplantation: radiologic evaluation, risk factors, and occult diagnosis. Hepatoma Res 2016;2:36-41. 


\section{INTRODUCTION}

In 1985, Shaw et al. ${ }^{[1]}$ reported the first successful cases of liver transplantation (LT) in the setting of recipient portal vein thrombosis (PVT). PVT was once considered an absolute contraindication to LT due to the considerable risk of intraoperative mortality and the technical difficulty of the operation. ${ }^{[2]}$ Advancements in operative technique, greater experience with the operation, and improved intra-operative anesthesia management have now made LT in patients with PVT increasingly common. ${ }^{[3]}$

It is estimated that the prevalence of PVT in cirrhotic patients who are the candidates for LT ranges from $5 \%$ to $26 \% .{ }^{[4]}$ Despite its prevalence, the understanding of PVT in the context of LT remains incomplete. Furthermore, the impact of PVT on the natural history and progression of cirrhosis is uncertain..$^{[5]}$ Although there is no clear evidence that PVT leads to further deterioration of liver function in advanced cirrhosis, this is often a common assumption or observation. Furthermore, PVT may be a source of technical difficulties in the particular setting of transplantation leading to a negative impact on outcomes. ${ }^{[4]}$ To date, the evidence regarding the effect of PVT on LT outcomes is mixed.

The mixed evidence exists regarding the risk factors for PVT, as well as the utility of preoperative imaging protocols in identifying the patients with, or at risk for PVT. ${ }^{[6-9]}$ As a result, it is estimated that more than $50 \%$ of patients with PVT remain undiagnosed until the time of surgery, even when a rigorous pre-operative screening protocol is utilized. ${ }^{[10,11]}$ In addition, the full extent of PVT is not evident until the LT operation..$^{7]}$ Since surgeons are unable to rely on imaging, pre-operative planning according to the severity of thrombosis remains difficult. However, as is the case with known PVT, it is still undetermined whether or how the occult, or incorrectly graded PVT, discovered at LT, impacts outcome.

Regarding the resource utilization in LT, it has been shown that longer length of stay (LOS) and higher cost of care are associated with increased severity of illness, increased number of procedures performed, and younger age. ${ }^{[12]}$ Resource utilization data specific to LT with PVT is limited. However, PVT has been associated with longer operative times and increased use of blood products. ${ }^{[13]}$

Herein, an analysis of the risk factors for PVT and independent predictors of survival were undertaken. We review the commonly used modalities for detection of PVT, and the effects of an uncertain pre-operative diagnosis of PVT on survival and resource utilization as determined by blood utilization/ transfusion rate [packed red blood cell (PRBC)], LOS, and postoperative morbidity at our institution.

\section{METHODS}

A retrospective analysis of 216 consecutive adult patients undergoing cadaveric LT from January 2007 to December 2013 at a single institution was undertaken. Patients with complete mesenteric venous thrombosis were excluded from LT, and all other patients were included in the analysis. Pre-operative patient demographics and clinical status were evaluated to identif y any potential risk factors for PVT. Routine imaging at our center consists of liver Doppler ultrasound (US) and a crosssectional imaging either a triple phase computed tomography (CT) or an Eovist magnetic resonance imaging (MRI). All imaging was reviewed by a multidisciplinary conference held weekly with all surgeons, hepatologists, body imaging radiologist, and interventional radiologists present. Interval imaging after listing a patient for transplantation consists of the US every 6 months. In patients with malignancy, contrasted CT, or MRI is done every 3 months until LT. The effectiveness of diagnosing PVT pre-LT, when PVT was later identified at LT, was evaluated for US, CT, MRI, and retrograde portal venography (RPV). Patient and graft survival were considered as primary endpoints. Blood utilization, LOS, and overall morbidity (Clavien grade II or greater) were used as surrogates of resource utilization. ${ }^{[14]}$ These were our secondary endpoints.

\section{Statistical analysis}

Continuous variables were compared between the groups using Student's t-test, categorical variables were compared using Chi-square test, and the serial values were compared using analysis of variance (ANOVA). Kaplan-Meier with log-rank analysis of actuarial patient and graft survival were calculated. LOS and PRBC were analyzed using ANOVA. Overall morbidity (Clavien grade II or greater) was compared between the groups by Chi-square analysis. Pre-operative characteristics that were significant on univariate analysis were evaluated by logistic regression to identify any potential risk factors for PVT. Multivariate survival analysis was done with a Cox proportional hazards model, and independent predictors of LOS and PRBC were analyzed by multivariate analysis of covariance.

\section{RESULTS}

Of 216 patients undergoing cadaveric LT, 30 (13.8\%) patients had PVT at the time of operation. Two hundred and five patients had at least one diagnostic imaging study within 1-year of LT. Only, 7 of 30 patients with PVT (23.3\%) had at least one positive imaging study suggestive of PVT pre-LT. The sensitivity of imaging techniques ranged $4.8-50 \%$, and the negative predictive value ranged $10.5-22.2 \%$ [Table 1].

Analysis of perioperative variables for those patients with and without PVT revealed that there was a significantly higher model for end-stage liver disease (MELD) score ( 25.0 vs. 21.4, $P=0.049$ ) and age (57.8 vs. 53.8, $P=0.041$ ) in those with PVT, although intensive care unit (ICU) status approached statistical significance (30\% vs. $15.6 \%, P=0.07$ ) [Table 2]. However, in our small series, the only factor by logistic regression that significantly predicted PVT was age $[P=0.037$; hazard ratio $(\mathrm{HR})=0.95]$.

Overall 90-day, the patient and graft survivals were $90.7 \%$ and 
Table 1: Pre-operative imaging studies

\begin{tabular}{lcccccc}
\hline Diagnostic study & Number of studies & Median days pre-LT & Sensitivity (\%) & NPV (\%) & Specificity (\%) & PPV (\%) \\
\hline US (no flow = PVT) & 149 & 26 & 4.8 & 13.8 & 97.7 & 25.0 \\
US (no, diminished, or & 149 & 26 & 31.6 & 10.5 & 85.4 & 24.0 \\
reversal of flow = PVT) & 158 & 56.5 & 19.0 & 11.2 & 98.5 & 66.7 \\
CT & 51 & 66 & 12.5 & 14.3 & 97.7 & 50.0 \\
MRI & 11 & 45 & 50.0 & 22.2 & 100.0 & 100.0 \\
RPV & & &
\end{tabular}

The efficacy of pre-operative diagnostic studies has long been questioned. Our data support this as well. Even when we set criteria for ultrasound diagnosis liberally (2nd US row), the sensitivity and NPV were wholly inadequate. Though the number is small, in our series even RPV, a direct and invasive technique only detected PVT pre-LT in half the cases. LT: liver transplant; PVT: portal vein thrombosis; NPV: negative predictive value; PPV: positive predictive value; RPV: retrograde portal venography; US: ultrasound

\begin{tabular}{llll}
\multicolumn{2}{l}{ Table 2: Variables related to PVT } \\
\hline Perioperative variables & PVT & No PVT & P \\
\hline Pre-operative variables & & & \\
Age & 57.8 & 53.8 & $\mathbf{0 . 0 4 1}$ \\
Gender: female & $23.3 \%$ & $33.3 \%$ & $\mathrm{NS}$ \\
Non-Caucasian race & $30.0 \%$ & $29.0 \%$ & $\mathrm{NS}$ \\
Medicare or medicaid & $20.0 \%$ & $43.0 \%$ & $\mathrm{NS}$ \\
Hepatocellular carcinoma & $2.8 \%$ & $13.6 \%$ & $\mathrm{NS}$ \\
diagnosis & & & \\
Hepatitis C virus diagnosis & $50.0 \%$ & $52.7 \%$ & $\mathrm{NS}$ \\
MELD & 25.0 & 21.4 & $\mathbf{0 . 0 4 9}$ \\
Cr & 1.6 & 1.8 & $\mathrm{NS}$ \\
Total bilirubin & 7.2 & 5.0 & $\mathrm{NS}$ \\
INR & 2.0 & 1.8 & $\mathrm{NS}$ \\
Pre-LT ICU status & $30.0 \%$ & $15.6 \%$ & 0.07 \\
Pre-LT hemodialysis & $26.7 \%$ & $15.6 \%$ & $\mathrm{NS}$ \\
Previous upper abdominal surgery & $30.0 \%$ & $25.8 \%$ & $\mathrm{NS}$ \\
Intra- and post-operative variables & & & \\
Cold ischemic time & 367.7 & 350.2 & $\mathrm{NS}$ \\
Warm ischemic time & 35.9 & 34.4 & $\mathrm{NS}$ \\
PRBC & 28.9 & 17.5 & $\mathbf{0 . 0 0 1}$ \\
Reentry & $40.0 \%$ & $36.0 \%$ & $\mathrm{NS}$ \\
Morbidity ( $\geq$ Clavien II) & $43.3 \%$ & $37.6 \%$ & $\mathrm{NS}$ \\
LOS, total (days) & 19.8 & 16.6 & $\mathrm{NS}$ \\
\hline
\end{tabular}

Age, MELD score, and the amount of blood loss were greater in patients who had PVT (bold print signifies significant values). The proportion of patients in the ICU with PVT was greater but only approached statistical significance. With logistic regression, the only pre-operative factor independently associated with PVT was age $(P=0.037 / \mathrm{HR}=0.95)$. Pre-LT: pre-liver transplant; LOS: length of stay; PVT: portal vein thrombosis; ICU: intensive care unit; MELD: model for end-stage liver disease; PRBC: packed red blood cell; HR: hazard ratio; NS: not significant

90.3\%, and 1-year were $83.7 \%$ and $83.3 \%$. The patient and graft survival were inferior in those with PVT [Figure 1a]. The divergence of both patient and graft survival occurred at approximately 6 months post-operatively. The patients with PVT at LT without a pre-LT diagnosis ("occult" PVT) did not have inferior patient or graft survival as compared to those with a definite pre-LT diagnosis $(P=0.79)$ [Figure $1 \mathrm{~b}]$. On multivariate analysis of patient survival, only MELD $>25(P=0.001, \mathrm{HR}=0.45)$ and age $>60$ years $(P=0.017, \mathrm{HR}=0.64)$ were independent risk factors for patient death. Similarly for graft survival, MELD $>25(P$ $=0.004, \mathrm{HR}=0.52)$ and age $>60$ years $(P=0.013, \mathrm{HR}$ $=0.67$ ) predicted graft loss independently [Table 3]. The presence of PVT diagnosed pre-LT or as an occult finding was not an independent predictor of either patient or graft survival on multivariate analysis.

LOS and PRBC requirements were also assessed. Although PRBC requirements were greater with PVT (28.9 vs. 17.5,

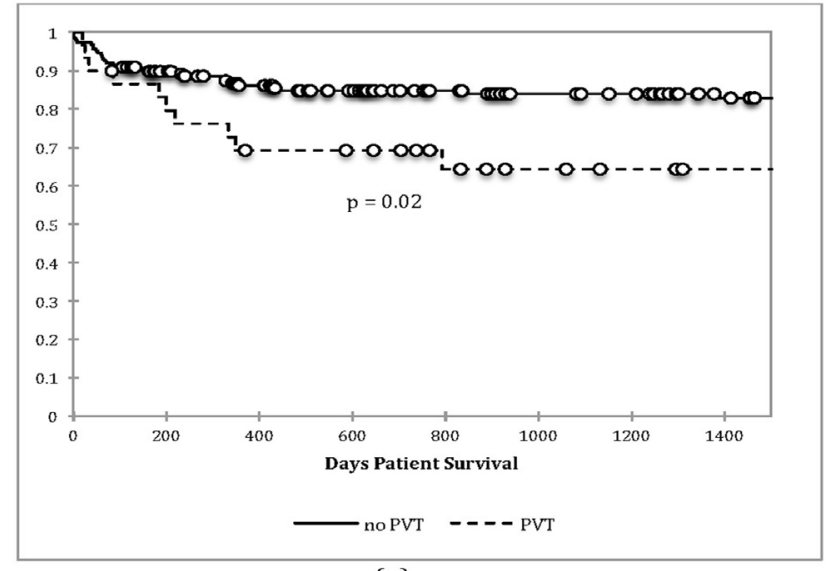

(a)

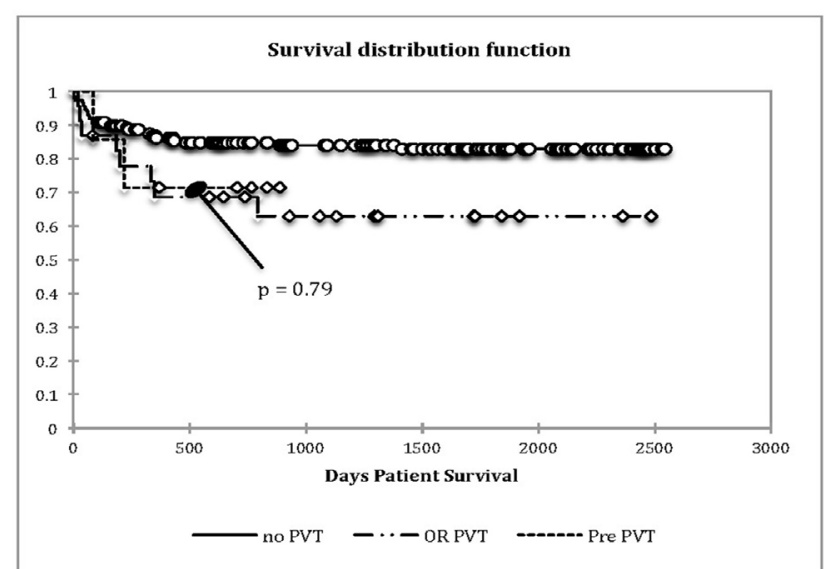

(b)

Figure 1: Patient survival and portal vein thrombosis (PVT). (a) PVT was associated with significantly reduced survival at approximately 6 months. Early perioperative survival (i.e., 90-day) was not significantly different, but divergence in survival occurred at 6 months; (b) there was no difference in survival between those with pre-liver transplantation diagnosis (prePVT) and those with "occult" PVT (OR PVT). Results for graft survival demonstrated the similar findings. On multivariate analysis [Table 3], PVT was not an independent predictor of survival

$P=0.001$ ), patients with PVT did not have a longer LOS (19.8 vs. $16.6, P=0.36)$ or greater morbidity $(43.3 \%$ vs. $37.6 \%, P>0.05)$ [Table 2]. Only PVT $(P=0.002)$ and pre-LT hemodialysis (HD) $(P=0.013)$ were significant covariates associated with increased PRBC [Figure 2]. When examining LOS, only female gender $(P=0.008)$, pre-LT HD $(P=0.012)$, and re-laparotomy $(P<0.0001)$ were significant at predicting the longer LOS [Figure 3]. 
Table 3: Analysis of patient and graft survival

\begin{tabular}{|c|c|c|c|c|c|c|}
\hline \multirow[t]{2}{*}{ Independent variable } & \multicolumn{2}{|c|}{ Patient survival } & \multirow[t]{2}{*}{ HR } & \multicolumn{2}{|c|}{ Graft survival } & \multirow[t]{2}{*}{ HR } \\
\hline & Univariate & Multivariate & & Univariate & Multivariate & \\
\hline Age $>60$ & 0.046 & 0.017 & 0.64 & 0.027 & 0.013 & 0.67 \\
\hline Gender: male & 0.144 & & & 0.102 & & \\
\hline Hepatitis C virus diagnosis & 0.52 & & & 0.76 & & \\
\hline Hepatocellular carcinoma diagnosis & 0.26 & & & 0.28 & & \\
\hline Private insurance & 0.138 & & & 0.244 & & \\
\hline Socioeconomically disadvantaged & 0.284 & & & 0.135 & & \\
\hline Pre-LT ICU stay & 0.015 & NS & & 0.023 & NS & \\
\hline Pre-LT hemodialysis & $<0.001$ & NS & & 0.001 & NS & \\
\hline Simultaneous kidney transplant & 0.04 & NS & & 0.045 & NS & \\
\hline Lab MELD > 25 & 0.02 & 0.001 & 0.49 & 0.03 & 0.004 & 0.52 \\
\hline PVT & 0.02 & NS & & 0.031 & NS & \\
\hline Occult PVT & 0.062 & & & 0.092 & & \\
\hline Complete PVT & 0.04 & NS & & 0.045 & NS & \\
\hline Past upper abdominal surgery & 0.236 & & & 0.331 & & \\
\hline PRBC $>20$ & 0.002 & NS & & 0.001 & NS & \\
\hline Reentry & 0.001 & NS & & 0.001 & NS & \\
\hline
\end{tabular}

All univariates were analyzed by Kaplan-Meier method with a log-rank test for significance. The significant univariates (bold print signifies significant values, $P<0.05$ ) were analyzed by a multivariate Cox's proportional hazards model to determine which independent predictors of survival. The only factors that appear to independently predict patient and graft survivals are advanced age $(>60)$ and advanced liver disease (MELD > 25). Interestingly, PVT was not an independent predictor of survival. Pre-LT: pre-liver transplant; MELD: model for end-stage liver disease; PRBC: packed red blood cell; PVT: portal vein thrombosis; ICU: intensive care unit; HR: hazard ratio; NS: not significant

\section{DISCUSSION}

The risk factors for PVT are often conflicting and not well established. Previously identified risk factors in historical patient series have included: Male gender, Child-Pugh class C disease, treatment for portal hypertension, variceal bleeding, abdominal surgery, as well as various etiologies of liver disease..$^{[7-10]}$ Conversely, age, sex, MELD score, treatment for portal hypertension, abdominal surgery, and etiology of liver disease have been identified as non-contributory factors in overlapping patient series. ${ }^{[7,9,10]}$ Such contradictory results highlight the need for further investigation to identify the independent risk factors associated with PVT.

Of 216 patients undergoing cadaveric LT, the prevalence of PVT in this center (13.8\%) fell within the expected range predicted by most historical series. Advanced age and perhaps higher MELD and ICU status were the risk factors for PVT in our series. It is possible that the duration and/or severity of portal hypertension seen in older patients with higher MELD scores contribute to PVT risk. The lack of statistical significance of higher MELD score and ICU status in predicting PVT on multivariate analysis may represent a type II statistical error. Furthermore, other factors such as a patient's sex, race, insurance status, diagnosis of hepatocellular carcinoma, hepatitis $\mathrm{C}$ virus, need for pre-LT HD, or surgical history did not contribute significantly to PVT risk in this study [Table 2]. These data, while relevant to this institution, do little to clear up the mixed picture of PVT risk factors overall, especially given the relatively small number of patients in this study. Further multicenter studies are clearly warranted.

In addition to examining the risk factors associated with PVT, we also attempted to examine the diagnostic capabilities for detecting PVT at our institution. Results from our series demonstrated that imaging was not effective at excluding PVT. The sensitivity and negative predictive values of various imaging modalities (US, CT, MRI, and RPV) in detecting PVT were poor [Table 1]. This is congruent with the results from the previous series, which have been demonstrated that the degree of PVT may be overestimated or underestimated, or it may be missed entirely by pre-operative imaging. ${ }^{[6,7]}$ It is estimated that more than $50 \%$ of patients with PVT remain undiagnosed until the time of surgery even with rigorous screening protocols in place. ${ }^{[10,11]}$ These high false negative rates are often attributed to the variability in the skill and experience of a US technicians and radiologists. ${ }^{[7]}$ Experience and preference of the radiologist greatly impact the quality of information obtained from any imaging study.

Missed diagnoses are most common in patients with partial $\mathrm{PVT},{ }^{[6,15]}$ although they have been described in those with complete thrombosis as well. ${ }^{[8]}$ In other patients, PVT is graded incorrectly, such that the full extent of thrombosis is not evident until the time of operation. ${ }^{[6]}$

The evidence regarding the impact of PVT on LT outcome is mixed, and whether an occult diagnosis has any additional effect on outcome is also uncertain. Using Kaplan-Meier survival curves, we found that the patient and graft survival were inferior in those with PVT and that the divergence of both patient and graft survival occurred at approximately 6 months following LT [Figure 1]. On multivariate analysis, MELD > 25 and age were significant independent predictors of patient and graft survival, while the presence of PVT was not. Age thus appears to be an independent predictor of PVT, as well as survival, and that survival is not predicated on the presence of PVT in this study. Furthermore, the discovery of PVT at 

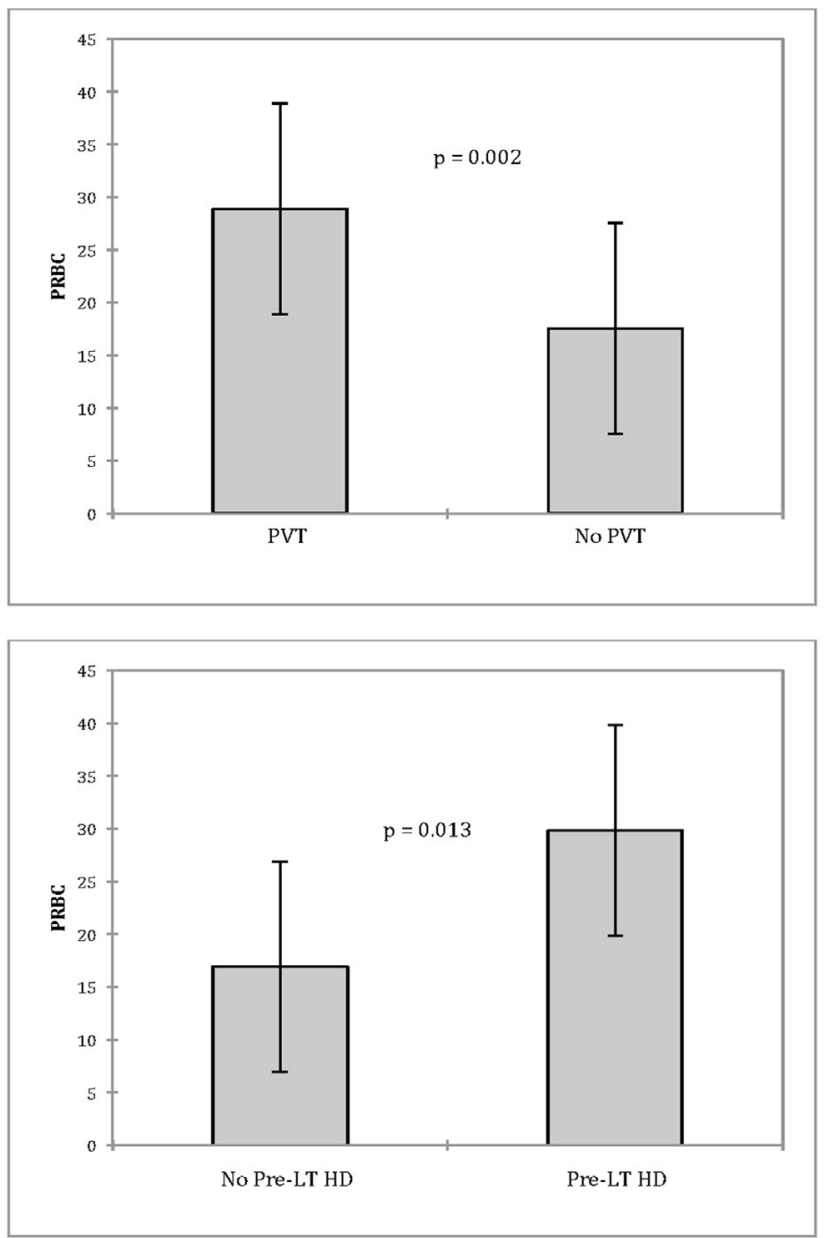

Figure 2: Predictors of PRBC utilization. Two independent predictors of blood utilization (PRBC) were identified by multivariate analysis of covariance: PVT and pre-LT HD. PRBC: packed red blood cell; PVT: portal vein thrombosis; Pre-LT: pre-liver transplant; HD: hemodialysis

the time of LT, without a pre-LT diagnosis ("occult" PVT), did not correlate with inferior patient or graft survival. Previous studies have also demonstrated that PVT does not have a significant effect on survival. ${ }^{[6,11,16,17]}$ The largest patient series to date, however, found that independent of MELD score; pre-transplant PVT was associated with up to a $50 \%$ increase in 1-year mortality post-transplant. ${ }^{[4,18]}$ Once again, the data in the literature is conflicting.

Survival in patients undergoing LT with PVT varies with the severity of thrombosis and the type of revascularization per formed..$^{[4,6]}$ When conventional end-to-end portal anastomosis can be achieved, whether PVT is partial or complete, results are comparable to LT recipients without PVT, with 1- and 5-year survival ranging from $84 \%$ to $86 \%$ and $65 \%$ to $80 \%$, respectively..$^{[4,6,11,16,19,20]}$ When alternative, non-anatomical revascularization techniques are necessary, such as renoportal anastomosis or cavoportal hemitransposition, survival is inferior with 1- and 5-year survival rates of only $60 \%$ and $38 \%$, as well as early post-operative mortality risks of $25 \%$. These techniques are typically reserved for the cases with extensive thrombosis involving the splenic or superior mesenteric veins and are only performed at a handful of centers. ${ }^{[21-23]}$ In our
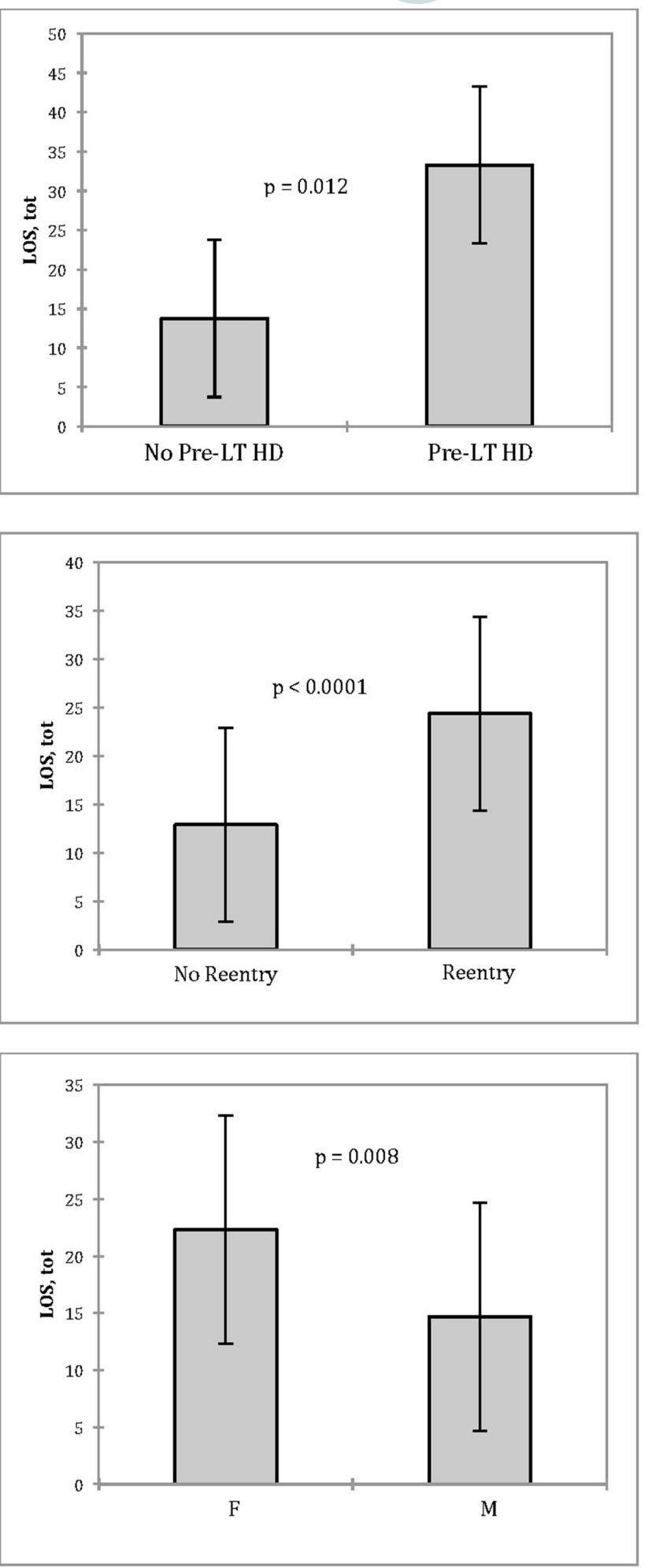

Figure 3: Predictors of LOS. Three independent predictors of prolonged LOS were identified by multivariate analysis of covariance: pre-LT HD, need for reentry, and female gender. LOS: length of stay; Pre-LT: pre-liver transplant; HD: hemodialysis

series, all patients with pre-operatively recognized extensive PVT of the entire portal venous system were excluded. Given the relatively small size of this patient series, and the conflicting data within the literature, the true effect of PVT on survival after LT remains incompletely understood. Further 
studies, along with a multicenter pooling and analysis of data would be a key to providing insight into this area.

Not only is PVT thought to increase post-transplant mortality, but morbidity has been argued by some to be affected as well. PVT has been associated with increased risk of sepsis, ${ }^{[6,22-25]}$ gastrointestinal bleeding due to persistent portal hypertension, ${ }^{[4,21,22,24,25]}$ ascites, renal dysfunction, ${ }^{[4,24]}$ and thrombotic events such as thrombosis at the anastomosis, hepatic artery, and pulmonary embolism. ${ }^{[24,25]}$ This may reflect the greater technical difficulty in the operation, the advanced disease state of patients with PVT, or a combination of the two. In our series, PVT was not an independent determinant of survival. Our data suggest that it is the advanced age and more advanced liver disease in patients with PVT that contributes to reduced patient and graft survival. Furthermore, we found that PVT was only associated with greater blood loss. Aside from blood loss, PVT did not contribute significantly to resource utilization, as measured by LOS or post-operative morbidity. ${ }^{[14]}$ These data are encouraging but need to be combined with larger patient series to establish more generalizable data.

In conclusion, neither patient or graft survival nor resource utilization in the form of LOS or overall post-operative morbidity was adversely affected by a known diagnosis or an occult finding of PVT in LT at our center. However, PVT at the time of LT did result in increased blood loss. Although this probably affected cost, it did not affect LOS or morbidity in our series. These results are reassuring given the poor sensitivity and negative predictive value of current imaging. The data from our study indicate that an extensive search for PVT may not be warranted, and imaging should be ordered only when otherwise indicated (e.g., malignancy surveillance and known malignancy). Clinical suspicion for PVT should be high in older patients, especially with a high MELD score and/ or ICU status. By optimization of the patient and anticipatory anesthesia care, the patient may benefit by a reduction in blood loss.

\section{Financial support and sponsorship} Nil.

\section{Conflicts of interest}

There are no conflicts of interest.

\section{REFERENCES}

1. Shaw BW Jr, Iwatsuki S, Bron K, Starzl TE. Portal vein grafts in hepatic transplantation. Surg Gynecol Obstet 1985;161:66-8.

2. Van Thiel DH, Schade RR, Starzl TE, Iwatsuki S, Shaw BW Jr, Gavaler JS, Dugas M. Liver transplantation in adults. Hepatology 1982;2:637-40.

3. Stieber AC, Zetti G, Todo S, Tzakis AG, Fung JJ, Marino I, Casavilla A, Selby RR, Starzl TE. The spectrum of portal vein thrombosis in liver transplantation. Ann Surg 1991;213:199-206.

4. Francoz C, Valla D, Durand F. Portal vein thrombosis, cirrhosis, and liver transplantation. J Hepatol 2012;57:203-12.

5. Garcia-Pagan JC, Valla DC. Portal vein thrombosis: a predictable milestone in cirrhosis? J Hepatol 2009;51:632-4.

6. Ravaioli M, Zanello M, Grazi GL, Ercolani G, Cescon M, Del Gaudio M, Cucchetti A, Pinna AD. Portal vein thrombosis and liver transplantation: evolution during 10 years of experience at the University of Bologna. Ann Surg 2011;253:378-84.
7. Yerdel MA, Gunson B, Mirza D, Karayalcin K, Olliff S, Buckels J, Mayer D, McMaster P, Pirenne J. Portal vein thrombosis in adults undergoing liver transplantation: risk factors, screening, management, and outcome. Transplantation 2000;69:1873-81.

8. Davidson BR, Gibson M, Dick R, Burroughs A, Rolles K. Incidence, risk factors, management, and outcome of portal vein abnormalities at orthotopic liver transplantation. Transplantation 1994;57:1174-7.

9. Lendoire J, Raffin G, Cejas N, Duek F, Barros Schelotto P, Trigo P, Quarin $\mathrm{C}$, Garay V, Imventarza O. Liver transplantation in adult patients with portal vein thrombosis: risk factors, management and outcome. $H P B$ (Oxford) 2007;9:352-6.

10. Francoz C, Belghiti J, Vilgrain V, Sommacale D, Paradis V, Condat B, Denninger MH, Sauvanet A, Valla D, Durand F. Splanchnic vein thrombosis in candidates for liver transplantation: usefulness of screening and anticoagulation. Gut 2005;54:691-7.

11. Dumortier J, Czyglik O, Poncet G, Blanchet MC, Boucaud C, Henry L, Boillot $\mathrm{O}$. Eversion thrombectomy for portal vein thrombosis during liver transplantation. Am J Transplant 2002;2:934-8.

12. Wai H, Stepanova M, Saab S, Erario M, Srishord M, Younossi ZM. Inpatient economic and mortality assessment for liver transplantation: a nationwide study of the United States data from 2005 to 2009. Transplantation 2014;97:98-103.

13. Suarez Artacho G, Barrera Pulido L, Alamo Martinez JM, Serrano DiezCanedo J, Bernal Bellido C, Marín Gomez LM, Padillo Ruiz J, Gómez Bravo MA. Outcomes of liver transplantation in candidates with portal vein thrombosis. Transplant Proc 2010;42:3156-8.

14. Agopian VG, Kaldas FM, Hong JC, Whittaker M, Holt C, Rana A, Zarrinpar A, Petrowsky H, Farmer D, Yersiz H, Xia V, Hiatt JR, Busuttil RW. Liver transplantation for nonalcoholic steatohepatitis: the new epidemic. Ann Surg 2012;256:624-33.

15. Cherqui D, Duvoux C, Rahmouni A, Rotman N, Dhumeaux D, Julien M, Fagniez PL. Orthotopic liver transplantation in the presence of partial or total portal vein thrombosis: problems in diagnosis and management. World J Surg 1993;17:669-74.

16. Molmenti EP, Roodhouse TW, Molmenti H, Jaiswal K, Jung G, Marubashi S, Sanchez EQ, Gogel B, Levy MF, Goldstein RM, Fasola CG, Elliott EE, Bursac N, Mulligan D, Gonwa TA, Klintmalm GB. Thrombendvenectomy for organized portal vein thrombosis at the time of liver transplantation. Ann Surg 2002;235:292-6.

17. Tao YF, Teng F, Wang ZX, Guo WY, Shi XM, Wang GH, Ding GS, Fu ZR. Liver transplant recipients with portal vein thrombosis: a single center retrospective study. Hepatobiliary Pancreat Dis Int 2009;8:34-9.

18. Englesbe MJ, Schaubel DE, Cai S, Guidinger MK, Merion RM. Portal vein thrombosis and liver transplant survival benefit. Liver Transpl 2010;16:999-1005.

19. Manzanet G, Sanjuán F, Orbis P, López R, Moya A, Juan M, Vila J, Asensi J, Sendra P, Ruíz J, Prieto M, Mir J. Liver transplantation in patients with portal vein thrombosis. Liver Transpl 2001;7:125-31.

20. Bertelli R, Nardo B, Montalti R, Beltempo P, Puviani L, Cavallari A. Liver a single transplant center. Transplant Proc 2005;37:1119-21.

21. Paskonis M, Jurgaitis J, Mehrabi A, Kashfi A, Fonouni H, Strupas K, Büchler MW, Kraus TW. Surgical strategies for liver transplantation in the case of portal vein thrombosis-current role of cavoportal hemitransposition and renoportal anastomosis. Clin Transplant 2006;20:551-62.

22. Selvaggi G, Weppler D, Nishida S, Moon J, Levi D, Kato T, Tzakis AG. Ten-year experience in porto-caval hemitransposition for liver transplantation in the presence of portal vein thrombosis. Am J Transplant 2007;7:454-60.

23. Gerunda GE, Merenda R, Neri D, Angeli P, Barbazza F, Valmasoni M, Feltracco P, Zangrandi F, Gangemi A, Miotto D, Gagliesi A, Faccioli AM. Cavoportal hemitransposition: a successful way to overcome the problem of total portosplenomesenteric thrombosis in liver transplantation. Liver Transpl 2002;8:72-5.

24. Yan P, Yan LN. Cavoportal hemitransposition in liver transplantation:a new technique. Hepatobiliary Pancreat Dis Int 2003;2:202-5.

25. Azoulay D, Adam R, Castaing D, Muresan S, Essomba A, Vibert E, Savier E, Smail A, Veilhan LA, Bismuth H. Liver transplantation with cavoportal or renoportal anastomosis: a solution in cases of diffuse portal thrombosis. Gastroenterol Clin Biol 2002;26:325-30. (in French) 